\begin{tabular}{|r|c|c|c|c|c|}
\hline Revista Clío América & ISSN: 1909-941X & Vol. 12 & No. 23 & enero - junio de 2018 & 62 - 72 \\
\hline \multicolumn{6}{|c|}{ D0I: $10.21676 / 23897848.2618$} \\
\hline
\end{tabular}

\title{
Evaluación de la lógica dominante del servicio para el caso de los seguros de automóviles en Colombia
}

\author{
Service dominant logic premises automobile insurance in Colombia
}

RESUMEN: El servicio es uno de los ejes fundamentales de toda organización, el cual está siendo evaluado continuamente por el cliente, generando en él vínculos relacionales de corto, mediano o largo tiempo. El sector asegurador no es ajeno a este escenario toda vez que los clientes viven momentos de servicio únicos e irrepetibles, en los que la empatía juega un papel importante para marcar la diferencia; por tanto, este trabajo tiene como propósito evaluar las premisas fundamentales de la lógica dominante del servicio con expertos de seguros de automóviles en Colombia, buscando identificar las premisas de mayor impacto e interpretar los concepto de co-creación y valor percibido para los expertos de seguros de automóviles en Colombia. El abordaje del problema se realiza desde el método Delphi, una técnica basada en preguntas acerca de la visión del futuro en donde los expertos en seguros brindan su opinión hasta lograr consenso y congruencia de lo que puede prospectarse para el sector asegurador. Los principales hallazgos muestran que el sector puede prestar un mejor servicio invirtiendo en desarrollos tecnológicos en donde los procesos sean agiles, claros respecto a las coberturas adquiridas y que acompañen permanente a sus asegurados.

Palabras clave: lógica dominante del servicio - sector asegurador seguro de automóviles - marketing de servicios.

JEL: LM310; G220

ABSTRACT: Service is one of the fundamental axes of any organization which is continuously being evaluated by costumers, generating in them relational links of short, medium or long term. For insurance sector this scenario is relevant also, as the customers' experiences are unique and one-off moments of service where empathy has an important role in making a difference; therefore, this work has as purpose to evaluate the fundamental premises of the Service-dominant logic with experts in automobile insurance in Colombia, seeking to identify the premises with the highest impact and to construe the co-creation and perceived value concepts from their perspectives. The methodological approach is made from Delphi method, a technique based on questions about the vision of the future, where insurance experts provide their opinions until they reach consensus and congruence on what can be prospected for the insurance sector. The main findings show that the sector can provide a better service by investing in technological developments, whereby the processes be agile and clear regarding the coverage acquired, and permanently accompany their insured.

Keywords: Service Dominant Logic - Insurance Sector - Automobile Insurance - Service Marketing.

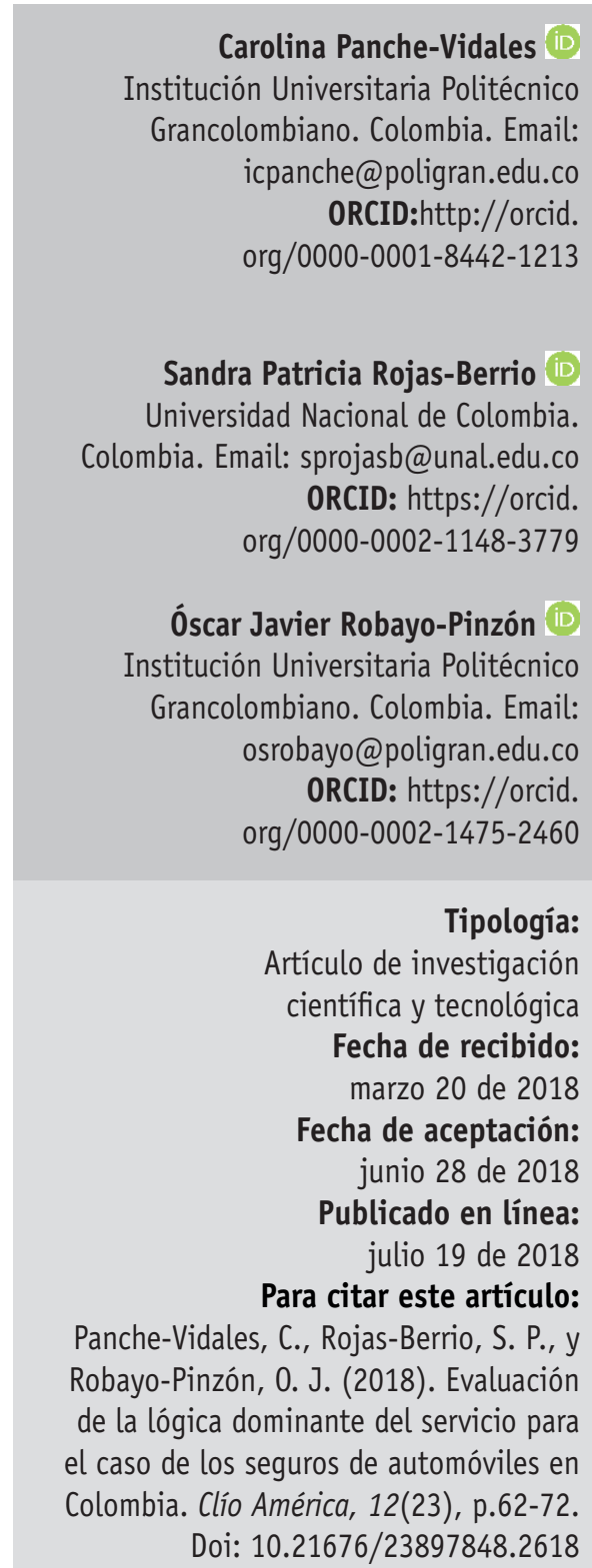




\section{INTRODUCCIÓN}

El servicio es un factor importante que, a pesar de ser invisible, determina la fidelización de los clientes de una organización (Grönroos y Gummerus, 2014). Adicionalmente, el servicio debe ser visto como una combinación de tres variables: calidad en el servicio (relacional y el valor percibido), satisfacción del cliente y futuras interacciones (McDougall y Levesque, 2000); estas variables deben crear valor a los clientes, como objetivo económico de toda compañía (Ng. y Smith, 2012). Así mismo, la literatura sobre este servicio presenta alternativas para abordarlo, que permiten revisar las competencias especializadas, conocimientos y habilidades que debe tener una organización que desee cumplir a cabalidad la promesa de servicio (Andrus y Hall, 2003; Ballantyne y Varey, 2008; Edvardsson, 2005; Grönroos, 2006), tales como la lógica dominante del servicio de Vargo y Lusch $(2004,2008)$.

En Colombia, el sector asegurador tiene una larga trayectoria protegiendo el patrimonio de los diferentes asegurados, brindando tranquilidad y respaldo por medio de soluciones intangibles, como lo son los seguros. Estos solo se vuelven tangibles en la medida en que se utilizan, por este motivo, el servicio es el protagonista de los diferentes momentos relacionados con esta operación. Actualmente se carece de estudios que investiguen las premisas fundamentales de la lógica dominante del servicio en los seguros, la banca 0 el sector financiero. Esta clase de estudios se han realizado para hoteles, restaurantes, clubes deportivos y para el sector médico, sectores en los que se ha avanzado en el ambiente de servicio (Bitner, 1992; Kim y Hardin, 2010; Perrone, 2009; García, Alvarez y Santos, 2011).

La Federación de Aseguradores Colombianos (Fasecolda) representa la actividad del sector asegurador, en su revista No. 163, de 2016, en la que indica que:

En Colombia la industria automotriz ha mostrado que a pesar de la caída de un $29 \%$ en la venta de vehículos nuevos, el aumento de variables como el número de vehículos asegurados $(5,1 \%)$ y de la prima promedio (4,7\%), esta última como consecuencia del incremento de los costos de reparación afectados por la revaluación del dólar, llevaron a un crecimiento del $10 \%$ de primas en el ramo (Silva y Nájera, 2016, p. 34).

Teniendo en cuenta lo anterior, las cifras muestran que el seguro de automóviles adquiere relevancia para las personas, para proteger su patrimonio, en relación con los riesgos que este pueda sufrir, tales como choques, robos o lesiones a terceros. El alto volumen de vehículos circulando en la ciudad hace que los accidentes y demás situaciones se incrementen, y que por este motivo se traslade el riesgo a una aseguradora, para sentir tranquilidad, seguridad y acompañamiento continuo (Vanegas y Varela, 2011).

Por otra parte, en Colombia, en 2014, aparece la más reciente reglamentación sobre la educación financiera, misma que es un derecho para los consumidores y una obligación para el sector financiero. A su vez, se cuenta con la transparencia del reporte de peticiones, quejas y reclamos de los asegurados, a través de los organismos de control y la agremiación: Superfinanciera y Fasecolda (Régimen de Protección al Consumidor Financiero, Ley 1328 de 2009 [Congreso de Colombia]). Por las anteriores razones, las aseguradoras, en Colombia, deben trabajar continuamente en las mejoras del servicio, cambiando un modelo servicio tradicional (desde la compañía hacia el cliente) por un servicio que escuche y se adecúe de acuerdo con las necesidades del cliente, y que se llama la co-creación del servicio; es decir, que las compañías trabajen conjuntamente con el cliente, interpretando sus necesidades, para entregar un producto que le genere valor, entregándole, en el caso de las aseguradoras, una póliza que pueda percibir como hecha a su medida, mejorando toda su experiencia en cualquier momento de encuentro con la empresa, de manera que el cliente, todo el tiempo, cree valor por su participación en los procesos, los cuales están en continua evaluación. (Grönroos y Gummerus, 2014; Payne, Storbacka y Frow, 2008; Payne, Storbacka, Frow y Knox, 2009; Vargo y Lusch, 2008). Esta evaluación también debe contemplar la parte humana de la interacción del servicio, a través del intercambio de información (Barile, Saviano y Polese, 2014). 
Si se considera que el ramo de automóviles creció al $10 \%$ de primas, en el año 2015 (Silva y Nájera, 2015), y también que los seguros de automóviles en Colombia representan el $14,5 \%$ de las primas totales de la industria aseguradora, se demuestra que este es de gran importancia para el sector asegurador (Vanegas y\& Varela, 2011). Por lo anterior, se puede afirmar que el seguro de automóviles es un servicio que está siendo utilizado en gran volumen, por lo que las compañías de seguros deben estar monitorizando las manifestaciones de inconformidad, a través de las quejas que reportan los usuarios de estas pólizas, con lo que se pueden identificar, analizar, medir y controlar los procesos que no están bien, y requieren ajustes durante la interacción con el servicio. Esta insatisfacción se genera cuando una experiencia se realiza fuera de la zona de aceptación del cliente. Si estas manifestaciones se tienen presentes se lograría convertir clientes decepcionados en clientes satisfechos, con efectos positivos de lealtad y retención. (Aarikka y Jaakkola, 2012; Tronvoll, 2007).

El implementar una lógica dominante del servicio (Flint, Lusch y Vargo, 2014; Vargo y Lusch, 2004) hace que se pueda crear valor, de acuerdo a las necesidades del cliente, y de cara a los procesos que intervienen con la compañía; desde la asesoría, en el momento de comprar la póliza, indicando las coberturas, precio, formas de pago, inspección del vehículo, entrega de la póliza, atención de un siniestro; y en general, todos los valores agregados, no solo al vehículo, sino también a las personas. Sin embargo, se carece de investigaciones que revisen las premisas de la lógica dominante del servicio (LDS) en el ámbito del sector asegurador; de hecho, el único trabajo en el sector financiero que aborda la LDS versa sobre finanzas públicas (Dza, Fisher y Gapp, 2013). En respuesta a esta carencia, este trabajo se orienta a partir de la siguiente pregunta de investigación: ¿cuáles son las premisas fundamentales de la lógica dominante del servicio más relevantes para los expertos del sector de seguros de automóviles en Colombia?; simultáneamente, los objetivos específicos son:
1. Identificar cuál de las premisas de la lógica dominante del servicio es la de mayor impacto para los expertos en seguros de automóviles en Colombia.

2. Interpretar los conceptos de co-creación y valor percibido, que hacen parte de las premisas fundamentales de la lógica dominante de servicio para los expertos de seguros de automóviles en Colombia.

Para todas las empresas y sectores, sin importar su naturaleza, el servicio es un factor fundamentalmente invisible, que determina que los clientes se fidelicen o terminen odiando una compañía (Bitner, Booms y Stanfield, 1990). En este sentido, Vargo y Lusch (2004) definen servicio, como: "la aplicación de competencias especializadas, conocimientos y habilidades, a través de hechos, procesos y funciones para el beneficio de otra entidad o la entidad en sí misma" (p. 3).

Adicionalmente, se debe entender, en primera instancia, que el cliente es el elemento central de toda compañía y que este tiene expectativas y percepciones del servicio prestado, a partir de las interacciones con empleados, proveedores, canales de comunicación y venta; en segunda instancia, que este agente está evaluando permanentemente el cumplimiento de la promesa de servicio (Andrus y Hall, 2003; Ballantyne y Varey, 2008; Edvardsson, 2005; Grönroos, 2006).

De igual forma, Vargo y Lusch (2004) proponen una perspectiva diferente para el marketing, evolucionando la lógica dominante de bienes a una lógica dominante de servicio, en donde el cliente juega un papel importante, a través de la interacción con la empresa; co-creando valor y co-produciendo los servicios que va a consumir. Teniendo en cuenta lo anterior, las empresas deben estar atentas a conocer lo que dicen los clientes sobre los productos o servicios ofrecidos, ya que ellos buscan un gana-gana, haciendo que las compañías crezcan o desaparezcan, con el tiempo.

\section{Lógica Dominante del Servicio}

Vargo y Lusch (2008) establecen diez premisas fundamentales de la Lógica dominante de Servicio, que se listan a continuación: 
- PF1: El servicio es la plataforma esencial de toda transacción.

- PF2: El servicio no siempre es evidente cuando, en las transacciones, existe una combinación de dinero, bienes e infraestructura.

- PF3: Los productos tangibles (perecederos y no perecederos) son elementos que ayudan percibir el servicio.

- PF4: Las habilidades y conocimientos son la base de una ventaja competitiva.

- PF5: Servicio es igual a riqueza.

- PF6: El valor no se produce se co-crea.
- PF7: Para que exista valor, se debe interactuar con el cliente la propuesta de valor.

- PF8: Un servicio enfocado al cliente implica la co-creación de un servicio relacional.

- PF9: Las compañías reúnen y transforman recursos.

- PF10: El cliente es quien califica el valor percibido.

Es relevante destacar que los autores también desarrollan una comparación entre lógica tradicional o lógica dominante de bienes, en contraste con la lógica dominante de servicio, misma que se representa en la Tabla 1.

Tabla 1.

Diferencias entre la Lógica Dominante de Bienes y la Lógica Dominante de Servicio

\begin{tabular}{|c|c|c|}
\hline & LÓGICA DOMINANTE DE BIENES & LÓGICA DOMINANTE DE SERVICIO \\
\hline $\begin{array}{l}\text { Unidad primaria de } \\
\text { intercambio }\end{array}$ & Las personas intercambian bienes. & $\begin{array}{l}\text { Las personas intercambian conocimientos } \\
\text { y habilidades para recibir beneficios. }\end{array}$ \\
\hline Papel de los bienes & $\begin{array}{l}\text { Las mercancías son el producto } \\
\text { final. Y quien las comercializa } \\
\text { varía sus condiciones. }\end{array}$ & $\begin{array}{l}\text { Las mercancías son trasmisores de } \\
\text { conocimiento: "productos" que son } \\
\text { utilizados creando valor para el cliente. }\end{array}$ \\
\hline Papel del cliente & $\begin{array}{l}\text { El cliente es el receptor de las } \\
\text { mercancías. }\end{array}$ & El cliente es un coproductor de servicio. \\
\hline $\begin{array}{l}\text { Determinación y } \\
\text { sentido de valor }\end{array}$ & $\begin{array}{l}\text { El valor de las mercancías lo } \\
\text { determina el fabricante. Este } \\
\text { bien es definido como valor de } \\
\text { intercambio. }\end{array}$ & $\begin{array}{l}\text { El valor percibido se determina por el valor } \\
\text { de uso del consumidor. }\end{array}$ \\
\hline $\begin{array}{l}\text { Interacción del } \\
\text { Cliente }\end{array}$ & $\begin{array}{l}\text { Los clientes están actuando para } \\
\text { crear operaciones con recursos. }\end{array}$ & $\begin{array}{l}\text { El cliente es un activo participante de } \\
\text { intercambios relaciones y coproducción. }\end{array}$ \\
\hline $\begin{array}{l}\text { Fuentes de } \\
\text { crecimiento } \\
\text { económico }\end{array}$ & $\begin{array}{l}\text { La riqueza se obtiene de recursos } \\
\text { tangibles y bienes excedentes, los } \\
\text { cuales se puede poseer, controlar } \\
\text { y producir. }\end{array}$ & $\begin{array}{l}\text { La riqueza es la aplicación del conocimiento } \\
\text { y habilidades para la transformación de } \\
\text { recursos. }\end{array}$ \\
\hline
\end{tabular}

Fuente: elaboración propia basada en Vargo y Lusch (2004). 
La lógica dominante del servicio es una estrategia empresarial para identificar o desarrollar ventajas competitivas, identificar clientes potenciales y desarrollar propuestas de valor para el cliente, satisfaciendo sus necesidades y creando una relación a largo plazo, co-creando servicios desde la perspectiva del cliente.

En correspondencia con lo anterior, los clientes evalúan todas las actividades durante, y después, de las interacciones con la compañía, a través de procesos cognitivos y emocionales (Edvardsson, 2005); entre tanto, las empresas deben alinear sus procesos de cara a los clientes, para convertirlas en propuestas de valor diferenciadoras, a través del servicio (Carbone y Haeckel, 1994); Esto debe lograrse teniendo en cuenta la percepción del consumidor de la atención recibida por el personal y del entorno físico del ambiente de servicio, asunto que también es llamado momento de verdad; y por último, de la experiencia vivida, también llamada pistas de la experiencia (Carbone y Haeckel, 1994; Vargo y Lusch, 2004). La literatura sobre el tema muestra cómo las experiencias se centran, principalmente, en las determinaciones de valor de los individuos, en diferentes contextos (Helkkula, Kelleher y Pihlström, 2012; Jaakkola, Helkkula y Aarikka, 2015). De igual manera, Baxter (2012) indica que las relaciones entre comprador y vendedor facilitan la co-creación de valor, en las las interacciones del servicio, tales como el compromiso, la confianza y la satisfacción.

\section{METODOLOGÍA}

Este trabajo adopta el abordaje del problema desde el método Delphi (Fefer, De-Urioste Stone, Daigle y Silka, 2016), el cual busca encontrar tendencias futuras por medio del consenso de expertos que analizan un problema; una interesante manera de conocer su opinión sobre las aplicaciones de la lógica dominante de servicio (Vargo y Lusch, 2014) en el sector asegurador, y cuya estrategia es obtener información proyectada hacia el futuro. La literatura relativa muestra que los métodos de orden proyectivo incluyen la técnica Delphi (Fefer et al., 2016; Kent y Saffer, 2014).

Una de las características de este método es que debe ser interactivo; que se debe preguntar a los expertos el problema en más de una ocasión. Por esta razón, se realizaron dos sesiones, con el fin de revalidar las respuestas: manteniendo en el anonimato a cada experto; evitando influenciadores negativos y manteniendo con ellos una retroalimentación controlada; destacando las respuestas más significativas y socializando las estadísticas del grupo. Se trabajó con la mediana de las respuestas, hasta alcanzar el criterio de estabilización y opinión consensual.

Con respecto al procedimiento correspondiente a este método, se inicia a través del planteamiento de una pregunta; para este caso, fue necesario plantear preguntas por cada premisa, las cuales fueron de carácter cerrado y abierto. Las preguntas cerradas fueron del tipo escala Likert, y su propósito fue validar la relevancia de cada uno de los componentes de las premisas, desde las perspectivas de los expertos; las abiertas permitían que los expertos dieran cuenta de qué cambios se tienen que dar en el sector asegurador para lograr el desempeño adecuado del sector, en cada premisa. Es relevante indicar que se estableció un grupo coordinador en gestión y marketing de servicios, quienes fueron los que validaron el instrumento (carta y documento) y la lista de expertos. En cuanto al número de expertos, no hay una forma para determinar cuál sería el número óptimo de participantes, pero, de acuerdo con los estudios realizados por Dalkey, Brown y Cochran, (1970), quienes indican que con un mínimo de siete expertos el error disminuye notablemente, se determinó que se aplicaría este método con ocho expertos del sector, con una experiencia mayor a diez años en el sector asegurador, que se destacaran por su idoneidad y amplio conocimiento de la estructura y características del sector asegurador colombiano y de los seguros de automóviles, con fundamentos jurídicos, técnicos y comerciales.

De acuerdo con el método Delphi, se invitó a estos expertos a que expresaran su opinión de cara al servicio futurista en el sector asegurador, con un enfoque hacia los seguros de automóviles, evaluando cada una de las premisas de la lógica dominante del Servicio (Vargo y Lusch, 2008). Se utilizó un instrumento virtual, el cual fue validado y aprobado por 
un grupo coordinador en gestión y marketing de servicios, que fue enviado a sus correos electrónicos.

Para el resultado de las preguntas cuantitativas, se realizó un análisis de cada una de las premisas, a través de correlaciones, con el fin de validar la opinión consensual de cada experto, y las preguntas abiertas se analizaron a través de palabras claves o códigos, que identificaron la idea principal del experto.

\section{RESULTADOS}

A continuación, se presentan los resultados del consenso entre los panelistas. Se obtuvo que, de las nueve premisas, siete tienen correlación positiva, reflejando el acuerdo entre los expertos del sector asegurador, las cuales fueron: i) el servicio como la plataforma esencial de toda transacción; ii)los productos son elementos que ayudan a percibir el servicio; iii) las habilidades y conocimientos son la base de una ventaja competitiva; iv) todas las economías son economías de servicio; v) para que exista valor, se debe interactuar con el cliente la propuesta de valor; vi) un servicio enfocado al cliente implica la co-creación de un servicio relacional; y vii) el cliente es quien califica el valor percibido.

Sin embargo, dos premisas arrojaron resultados diferentes; una de ellas es la premisa dos, que habla del intercambio indirecto, el cual oculta la unidad principal de cambio. Analizada con las demás premisas, dio como resultado que solo un experto califica en cuadrante negativo las

correlaciones; los demás expertos califican en cuadrante positivo esta premisa, relacionada con las demás, tal como se presenta en la Figura 1.

Figura 1.

Asociación entre premisas 2 y 7

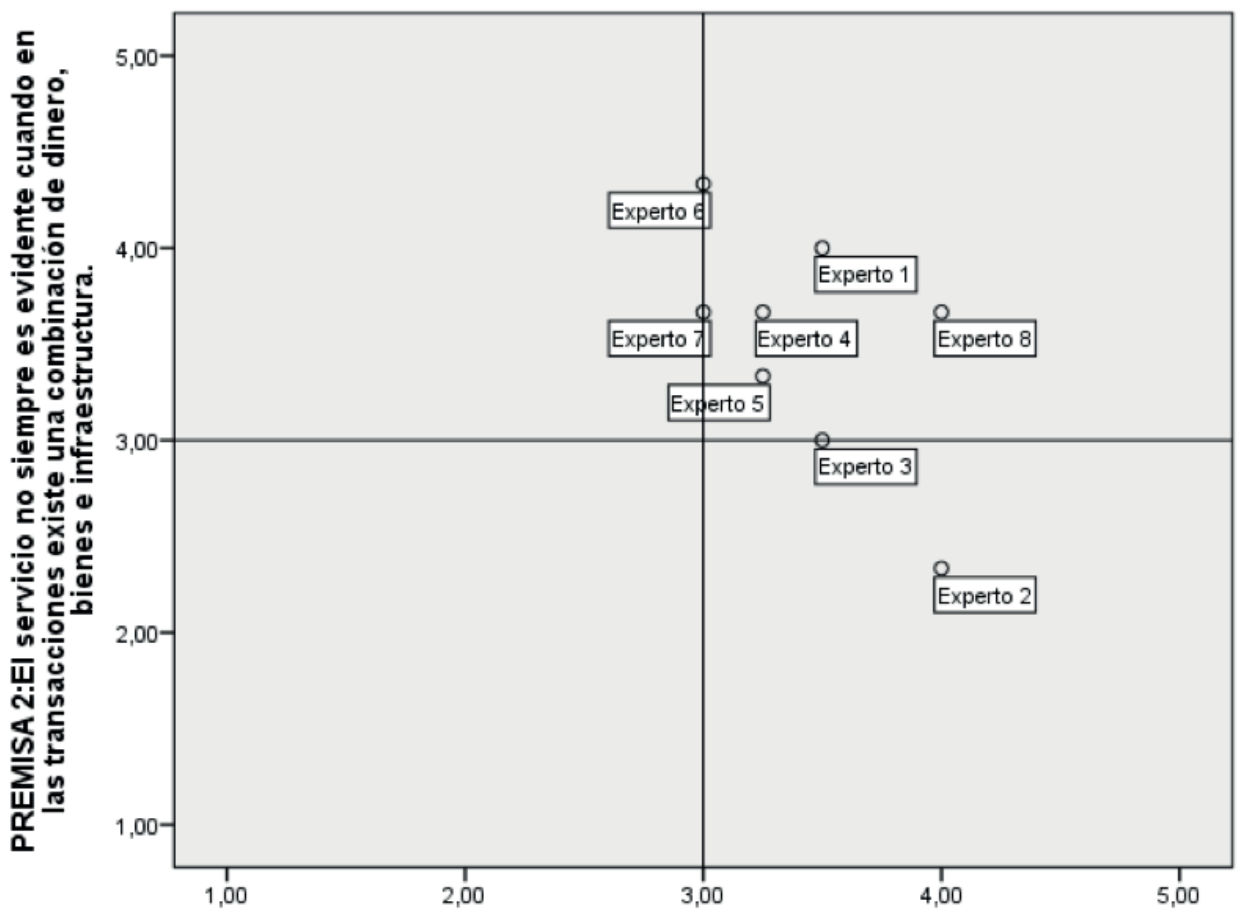

PREMISA 7: Para que exista valor se debe interactuar con el cliente la propuesta de valor

Fuente: elaboración propia. 
Un ejemplo de este resultado es la correlación de la premisa seis (El valor no se produce, se co-crea), con la premisa cinco (Todas las economías son economías de servicio), como se expone en la Figura 2.

La premisa seis, que afirma que el valor no se produce, se co-crea, es calificada por siete de los expertos en cuadrante negativo, resaltando su desacuerdo en la correlación de esta premisa con las demás, para el sector asegurador.

Adicionalmente, se les pidió a los expertos que indicaran: de lo más importante a lo menos importante, tres opiniones proyectadas al futuro sobre lo que deberían hacer en el sector asegurador, por cada premisa, con el fin de continuar evolucionando en el servicio, arrojando como resultado lo que se presenta en la Figura 3.

Un resultado interesante es que los expertos opinaron que las Apps, en el sector asegurador, ayudarían a percibir de una mejor manera el servicio, con procesos más agiles y sencillos para hacer uso de las coberturas de las pólizas, tal como se expone en la Figura 4.

Evaluando, en general, todas las opiniones de los expertos, las palabras claves a desarrollar en el sector asegurador son:

- Productos en línea.

- Procesos Virtuales - Apps.

- Mejorar tiempos.

- Capacitación.

- Servicio Personalizado.

Cabe anotar que, para lograr lo anterior, se encontró como limitación que los expertos son personas muy ocupadas, que no contaban con mucho tiempo para dedicarle a este estudio. Por esta razón, se otorgó un reconocimiento simbólico a través de un bono para compra de libros, para motivar la participación.

\section{Figura 2.}

\section{Asociación entre premisas 6 y 5}

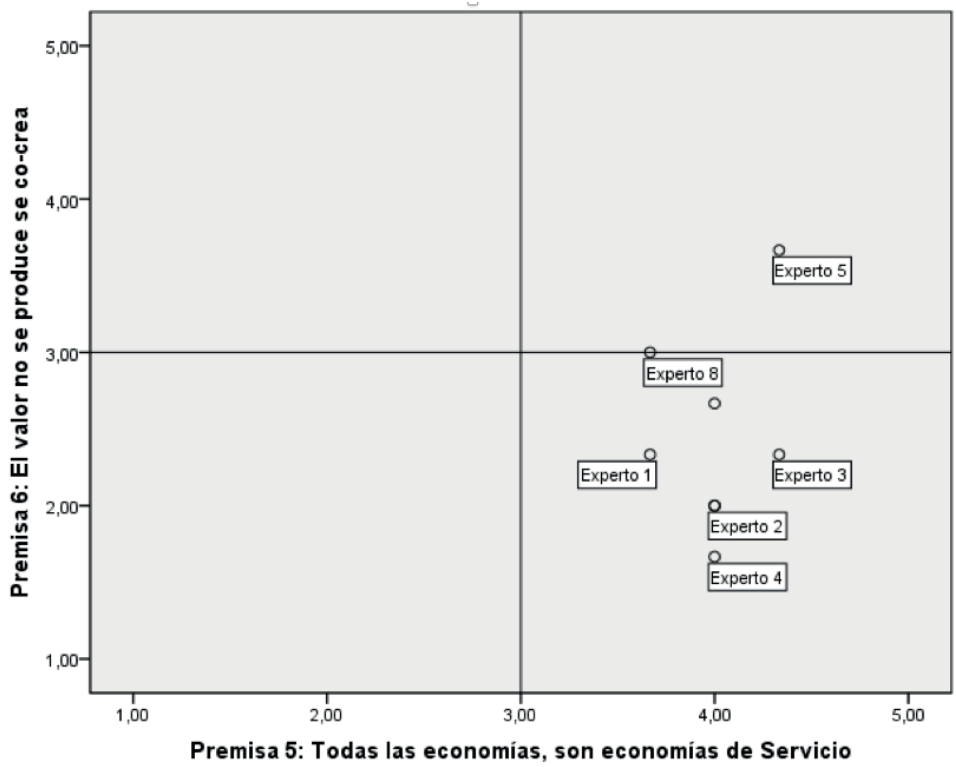

Fuente: elaboración propia. 
Figura 3.

Cambios en tecnologías y procesos que deberían ser abordados por el sector.

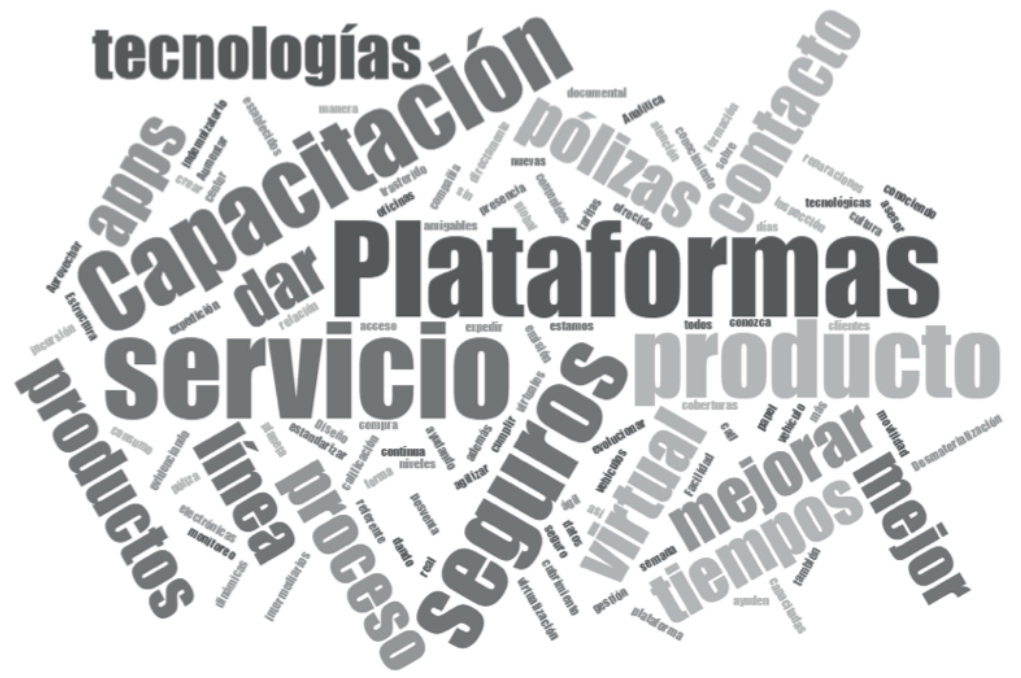

Fuente: elaboración propia.

Figura 4.

Resultados generales de las opiniones futuristas de los expertos

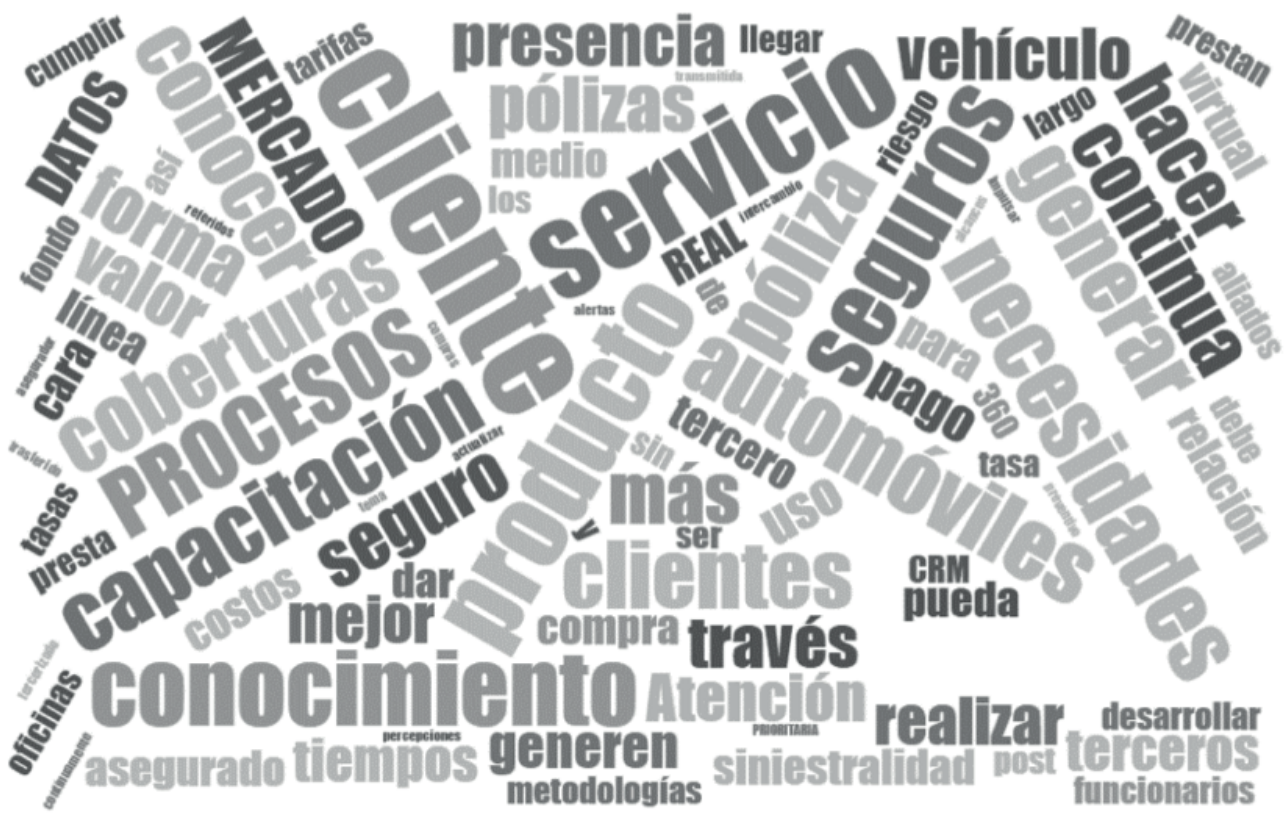

Fuente: elaboración propia. 


\section{DISCUSIÓN}

Las premisas de la lógica dominante del servicio, vistas desde el sector asegurador, aportarían positivamente para que el cliente de seguros de automóviles perciba de manera tangible los servicios diseñados, y para brindar tranquilidad y acompañamiento constante al asegurado.

Cuando se evalúan las premisas, los expertos identifican cuáles son las de mayor relevancia en el futuro. Una de ellas es la habilidad y conocimientos que hacen una ventaja competitiva fundamental en el sector asegurador, incluyendo procesos, coberturas, indemnizaciones, exclusiones, entre otros, que generan confianza al asegurado, en referencia a la compañía de seguros que lo ampara.

Los expertos concuerdan en que el servicio es esencial, y en que, para que el cliente valore dicho servicio, se debe interactuar con él la propuesta de valor, a través de plataformas de servicio que tengan conectividad en todo momento.

A través de este estudio, se puede determinar que el sector asegurador, en toda su estructura de servicio, debe tener una evolución, como lo plantea cada una de las premisas de la lógica dominante del servicio (Vargo y Lusch, 2016); en este caso, para el seguro de automóviles, ya que este seguro es el más comprado en Colombia.

Para el marketing de servicios, la lógica dominante (Vargo y Lusch, 2008) debe continuar evolucionando en diferentes sectores, como el asegurador, en la forma en la que se atienden las necesidades de sus asegurados, y a través de las mismas, generar relaciones a largo plazo.

\section{CONCLUSIÓN}

El inicio de esta investigación abre un camino para continuar evaluando las premisas de la lógica dominante del servicio con los usuarios finales de seguros de automóviles, si las premisas que consideran los expertos son las mismas para los asegurados, y de esta forma, permite identificar la brecha entre quienes conocen los seguros y quienes los utilizan. Adicionalmente, la evaluación de estas premisas se puede extender a otros seguros, como los de vida, hogar, empresarial, entre otros, si tienen el mismo comportamiento que los seguros de automóviles, de cara al servicio.

Se puede concluir que las premisas de la lógica dominante son aplicables para continuar mejorando el servicio en el sector asegurador, identificando que no hay una premisa que tenga mayor impacto para los expertos en seguros de automóviles, y resaltando las siguientes: i) los seguros de automóviles ayudan a percibir el servicio, independientemente de quien preste dicho servicio, ya sea, directamente una aseguradora o sus proveedores; ii) cuando se reconoce que el servicio es fundamental en toda transacción, a través de habilidades y conocimientos, estos generan una ventaja competitiva en el sector asegurado.

Al interpretar con los expertos el concepto de cocreación en seguros de automóviles, obtuvimos como resultado que esta es la única premisa que no es viable en el sector asegurador, puesto que la cocreación de un producto a la medida y necesidades del cliente, requiere de un producto personalizado, según el tipo de vehículo, la ciudad donde transita, las asistencias particulares, entre otros factores, los cuales, técnicamente, las compañías de seguros no pueden respaldar individualmente, sino a través de necesidades colectivas, a partir de las cuales las pólizas son evaluadas, diseñadas y tarifadas por cada aseguradora, de acuerdo al comportamiento de siniestralidad de los mismos; estas coberturas son validadas y aprobadas por el ente que las vigila, como la Superintendencia Financiera de Colombia. Por esta razón, no se puede construir un producto de seguros de automóviles, directamente con el asegurado.

También se evidenció que, si el cliente interactúa con lo que se le prometió, así mismo, el asegurado calificará el valor que percibe del mismo, co-creando con él un servicio relacional. 


\section{REFERENCIAS BIBLIOGRÁFICAS}

Aarikka-Stenroos, L. y Jaakkola, E. (2012). Value cocreation in knowledge intensive business services: $\mathrm{A}$ dyadic perspective on the joint problem solving process. Industrial Marketing Management, 41(1), 15-26. https://doi.org/10.1016/j.indmarman.2011.11.008

Andrus, D. M. y Hall, C. (2003). Services marketing: integrating customer focus across the firm (3rd ed.). Boston: McGraw-Hill/Irwin.

Ballantyne, D. y Varey, R. (2008). The service-dominant logic and the future of marketing. Journal of the Academy of Marketing Science, 36(1), 11-14. https:// doi.org/10.1007/s11747-007-0075-8

Barile, S., Saviano, M. y Polese, F. (2014). Information asymmetry and co-creation in health care services. Australasian Marketing Journal, 22(3), 205-217. https://doi.org/10.1016/j.ausmj.2014.08.008

Baxter, R. (2012). Chapter 5 Availability of Resources through Buyer-Seller Relationships. En M. S. Glynn y A. G. Woodside (Ed.), Business-to-Business Marketing Management: Strategies, Cases and Solutions. Advances in Business Marketing and Purchasing, 18, (pp. 119-140). Boston, USA: Emerald Group Publishing Limited. Recuperado de https://doi. org/10.1108/S1069-0964(2012)0000018010

Bitner, M. (1992). Servicescapes: the impact of physical surroundings on customers and employees. Journal of Marketing, 56(2), 57-71. https://doi. org $/ 10.2307 / 1252042$

Bitner, M., Booms, B. y Stanfield, M. (1990). The service encounter: Diagnosing favorable and unfavorable incidents. Journal of Marketing, 54(1), 71-84. https:// doi.org/10.2307/1252174

Carbone, L. y Haeckel, S. (1994). Engineering customer experiences. Marketing Management, 3(3), 8. Recuperado de https://www.researchgate.net/ publication/265031917_Engineering_Customer_ Experiences

Dalkey, N., Brown, B. y Cochran, S. (1970). The Delphi Method, III: Use of self rating to improve group estimates. Recuperado de https://www.rand.org/content/dam/ rand/pubs/research_memoranda/2006/RM6115.pdf

Dza, M., Fisher, R. y Gapp, R. (2013). Service-dominant logic and procurement in Africa: Lessons learned from a development agenda in ghana. International Journal of Economic Policy in Emerging Economies, 6(2), 141157. https://doi.org/10.1504/IJEPEE.2013.055794
Edvardsson, B. (2005). Service quality: beyond cognitive assessment. Managing Service Quality: An International Journal, 15(2), 127-131. https://doi. org/10.1108/09604520510585316

Fefer, J., Urioste de, S., Daigle, J. y Silka, L. (2016). Using the Delphi technique to identify key elements for effective and sustainable visitor use planning frameworks. SAGE Open, 6(2). https://doi. org/10.1177/2158244016643141

Flint, D., Lusch, R. y Vargo, S. (2014). The supply chain management of shopper marketing as viewed through a service ecosystem lens. International Journal of Physical Distribution and Logistics Management, 44(1), 23-38. https://doi.org/10.1108/ IJPDLM-12-2012-0350

García, N., Álvarez, B. y Santos, M. (2011). Aplicación de la lógica dominante del servicio (LDS) en el sector turístico: el marketing interno como antecedente de la cultura de co-creación de innovaciones con clientes y empleados. Cuadernos de Gestión, 11(2), 53-75. https://doi.org/10.5295/cdg.100238ng

Grönroos, C. (2006). Adopting a service logic for marketing. Marketing Theory, 6(3), 317-333. https://doi. org/10.1177/1470593106066794

Grönroos, C. y Gummerus, J. (2014). The service revolution and its marketing implications: Service logic vs service-dominant logic. Managing Service Quality, 24(3), 206-229. https://doi.org/10.1108/ MSQ-03-2014-0042

Helkkula, A., Kelleher, C. y Pihlström, M. (2012). Practices and experiences: challenges and opportunities for value research. Journal of Service Management, 23(4), 554570. https://doi.org/10.1108/09564231211260413 Jaakkola, E., Helkkula, A. y Aarikka-Stenroos, L. (2015). Service experience co-creation: conceptualization, implications, and future research directions. Journal of Service Management, 26(2), 182-205. https://doi. org/10.1108/J0SM-12-2014-0323

Kent, M. y Saffer, A. (2014). A Delphi study of the future of new technology research in public relations. Public Relations Review, 40(3), 568-576. https://doi. org/10.1016/j.pubrev.2014.02.008

Kim, J. y Hardin, A. (2010). The impact of virtual worlds on word-of-mouth: Improving social networking and servicescape in the hospitality industry. Journal of Hospitality Marketing and Management, 19(7), 735-753. https://doi.org/10.1080/19368 623.2010 .508005 
McDougall, G. y Levesque, T. (2000). Customer satisfaction with services: putting perceived value into the equation. Journal of Service Marketing, 14(5), 392-410. https://doi.org/10.1108/08876040010340937

Ng., I. y Smith, L. (2012). An Integrative Framework of Value. En S. L. Vargo y R. F. Lusch (Ed.), Special Issue - Toward a Better Understanding of the Role of Value in Markets and Marketing. Review of Marketing Research, 9, (pp. 207-243). Boston, USA: Emerald Group Publishing Limited. Recuperado de https://doi. org/10.1108/S1548-6435(2012)0000009011

Payne, A., Storbacka, K. y Frow, P. (2008). Managing the co-creation of value. Journal of the Academy of Marketing Science, 36(1), 83-96. https://doi. org/10.1007/s11747-007-0070-0

Payne, A., Storbacka, K., Frow, P. y Knox, S. (2009). Co-creating brands: Diagnosing and designing the relationship experience. Journal of Business Research, 62(3), 379-389. https://doi.org/10.1016/j. jbusres.2008.05.013

Perrone, A. (2009). How servicescape enhances service quality. International Journal of Knowledge, Culture and Change Management, 9(2), 103-111. https://doi.org/10.18848/1447-9524/CGP/ v09i02/49696

Regimen de Protección al consumidor Financiero (Ley 1328 DE 2009). Diario Oficial No. 47.411 de 15 de julio de 2009.

Silva, J. y Nájera, A. (015). Resultados de la industria aseguradora al mes de septiembre del 2015. Revista Fasecolda, (161), 66-71. Recuperado de https:// revista.fasecolda.com/index.php/revfasecolda/ article/view/189
Silva, J. y Nájera, A. (2016). Resultados de la industria aseguradora en el año 2015. Revista Fasecolda, (163), 34-41. Recuperado de http://www.fasecolda.com/ files/3614/6548/0848/revistaFasecolda163.pdf

Tronvoll, B. (2007). Customer complaint behaviour from the perspective of the service-dominant logic of marketing. Managing Service Quality, 17(6), 601-620. https://doi.org/10.1108/09604520710834966

Vanegas, V. y Varela, C. (2011). Seguro de daños. Seguro de Automóviles: Evolución y Comportamiento del Ramo de Automóviles. En Fasecolda (Ed. de la serie), La Industria Aseguradora en Colombia: Tomo II (pp. 352-395). Recuperado de http://www.fasecolda. com/files/4413/9101/6737/seguro_de_automoviles.pdf

Vargo, S. y Lusch, R. (2004). Evolving to a New Dominant Logic for Marketing. Journal of Marketing, 68(1), 1-17. https://doi.org/10.1509/jmkg.68.1.1.24036

Vargo, S. y Lusch, R. (2008). Service-dominant logic: Continuing the evolution. Journal of the Academy of Marketing Science, 36(1), 1-10. https://doi. org/10.1007/s11747-007-0069-6

Vargo, S. y Lusch, R. (2014). Inversions of servicedominant logic. Marketing Theory, 14(3), 239-248. https://doi.org/10.1177/1470593114534339

Vargo, S. y Lusch, R. (2016). Institutions and axioms: an extension and update of service-dominant logic. Journal of the Academy of Marketing Science, 44(1), 5-23. https://doi.org/10.1007/s11747-015-0456-3 